plants, food), and the discussion of modern materials and devices. Educational formats depend on the target audience and desired outcome.

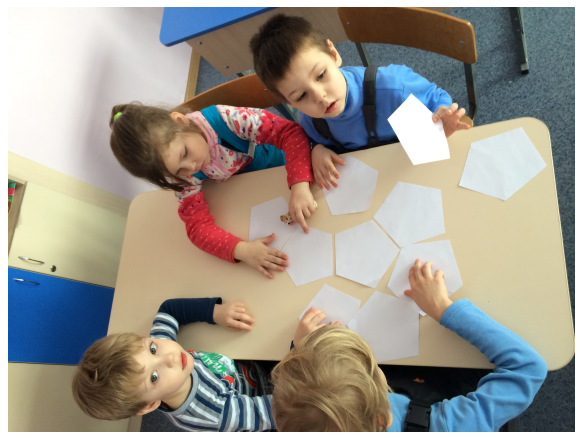

Figure 1. Tiling - an art or science? Keywords: public engagement, school education,
multi-disciplinary teaching

\section{MS47-O2 A cross-field perspective on Symmetry \& Crystal to introduce Crystallography}

Jean-Louis Hodeau ${ }^{1}$, Christophe Bouchard ${ }^{1}$, Sabine Douillet ${ }^{1}$, Florence Fernandez ${ }^{1}$, Laurent Joubert ${ }^{1}$, Yvonne Soldo ${ }^{1}$, Stephanie Zaccaro $^{1}$

1. Institut Neel, CNRS \& Univ. Grenoble Alpes, 38042 Grenoble, France

email: hodeau@neel.cnrs.fr

Crystallography uses two important tools, a concrete object: crystal, and an abstract description: symmetry. To illustrate the science of crystallography to the public, examples using the symmetry and crystal will be illustrated.

Symmetry as proportion and light played an important role in aesthetics (symmetry means "right measures / proportions" in Greek). Vitruvius developed the virtues of proportion and symmetry in architecture and in nature, as shown by Leonardo da Vinci in its "human body" drawing. Architecture makes extensive use of symmetry: symmetry is present in Islamic mosques, Chinese pagodas, Hindu temples and Gothic cathedrals. Tiling patterns are also a great example of symmetry, as shown in Islamic cultures in the Middle Ages.

Crystals shapes and facets comply geometric rules. Studying their symmetries allows us to differentiate the crystals. In the 19th century German and French researchers developed symmetry to classify all the crystals. They introduce the concepts of lattice, axis, center, and mirror plane of symmetry. Crystallographers have given a new meaning to the word symmetry itself, which had hitherto been reserved for architectural purposes. This classification of crystals according to their symmetry and lattice structure is still with us today. The symmetry links the shape of the crystals to their atomic structure and it is important for studying the physical properties of crystals. This abstract relationship between crystal and symmetry was confirmed in 1912, together with the periodicity of the crystal, by the first X-ray diffraction experiments.

We can use the crystal and the symmetry to focus public interest on crystallography and to show children and students the importance of observations, models and representations in scientific approaches. As an illustration, we will present several workshops using the facilities of Physiquarium and KaleidoLab. The capacity of KaleidoLab to generate models is used for the creation of "on-live" wallpapers and videos.

This presentation is the result of different actions: Voyage dans le Cristal exhibition (*) Krystallopolis website $(* *)$ and KaleidoLab workshop (***) and we thank colleagues who contributed to these actions, Marc DeBoissieu, Dominique Cornuejols, Thibaut David, Yannick Lacaze, René Guinebretière.

* http://www.iycr2014.org/resource-materials/voyage

** http://www.krystallopolis.fr/

****

http://neel.cnrs.fr/IMG/KaleidoLab_Symetrie-cest-quoi_14dec15-ss-s 


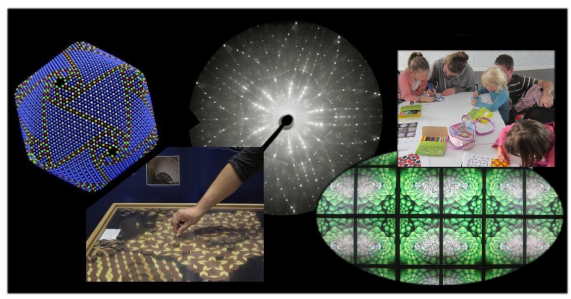

Figure 1. Children can play with mosaics to cover surface with identical shapes. These tiles with different symmetries allow the public to discover symmetry rules. The shape of the tiles is related to the symmetry of the lattice. (C) IUCrJournals \& KaleidoLab; Hodeau \& Guinebretiere, JAC 48, 1276 (2015)

Keywords: Crystallography, Teaching, Education, History

\section{MS47-O3 The power of using 827,948 crystal structures in education \\ Suzanna C. Ward ${ }^{1}$, Amy A. Sarjeant ${ }^{2}$}

1. The Cambridge Crystallographic Data Centre, 12 Union Road, Cambridge, CB2 1EZ, UK

2. The Cambridge Crystallographic Data Centre, Piscataway, NJ, 08854, USA

email: ward@ccdc.cam.ac.uk

The Cambridge Structural Database (CSD) contains over 800,000 experimentally determined small molecule crystal structures and this wealth of structural data can be a powerful tool in education. As well as providing the three dimensional structure of molecules, the CSD contains statistical information about symmetry, packing, coordination environments, bond distributions, and intermolecular interactions together with associated information such as physical properties and crystallization conditions.

This talk will focus on how structural data can be used in chemical education by using specific examples to illustrate how teachers can use the CSD to demonstrate key chemical concepts and principles. Students can learn a lot from this collection of structures, but given the vastness of the CSD identifying key structures that are most appropriate for a class is not always a simple process. We will therefore demonstrate, using examples, how the CSD teaching database, compiled to represent a diverse range of chemistry, can be used in the classroom to augment the learning experience of students in schools and universities worldwide.

Finally, we will reflect on the structural chemistry resources that are already available to educators and explore how these might evolve over the next 50 years of the CSD.

Keywords: Chemical Education, Teaching, Cambridge Structural Database, Structural Chemistry 